\title{
Análisis estructuralista de la teoría de la anomia*
}

Structuralist Analysis of Anomie Theory

Cláudio Abreu ${ }^{\dagger \neq}$

\begin{abstract}
Resumen
Si bien es posible encontrar el concepto de anomia en el pensamiento griego, es a partir de Durkheim que el concepto empieza a ser utilizado específicamente como un concepto sociológico. Sin embargo, una teoría de la anomia se consolida recién a partir de "Social Structure and Anomie", de Robert K. Merton (Merton 1938). La teoría se vuelve importante y conquista su espacio en el resto del siglo XX destacándose como una de las más productivas teorías acerca de la desviación. En este estudio, con base en una concepción contemporánea de teoria científica, la de la metateoría estructuralista, es presentada una reconstrucción de la propuesta de Merton, lo que permite explicitar la estructura profunda de la teoría.
\end{abstract}

Palabras clave: filosofía de la ciencia - metateoría estructuralista - Robert K. Merton - teoría de la anomia

\begin{abstract}
Although we may find the concept of anomie in Greek thought, it is since Durkheim that the concept begins to be used specifically as a sociological concept. However, a theory of anomie only becomes consolidated since "Social Structure and Anomie" by Robert K. Merton (Merton 1938). The theory becomes important and conquers its space in the rest of the century as one of the most productive theories about deviance. In this study, based on a contemporary conception of scientific theory, that of the structuralist metatheory, a reconstruction of Merton's approach is proposed, allowing to make explicit the deep structure of the theory.
\end{abstract}

Keywords: philosophy of science - metatheoretical structuralism - Robert K. Merton - anomie theory

* Recibido: 12 de Enero de 2014. Aceptado en: 27 de Marzo de 2014.

† Facultad de Guararapes, Brasil; Universidad Nacional de Quilmes, Argentina. Para contactar al autor, por favor escriba a: claudioabreu@outlook.com.

* Este trabajo ha sido realizado con la ayuda de los proyectos de investigación PICT2007 № 1558 (ANPCyT, Argentina) y PIP No 112 201101-01135 (CONICET, Argentina). Agradezco a los integrantes del seminario permanente de filosofía de la ciencia impartido en el marco del Programa de Investigación "Filosofía e Historia de la Ciencia" del Instituto de Estudios sobre la Ciencia y la Tecnología/Universidad Nacional de Quilmes, por las discusiones acerca de versiones previas del trabajo. Asimismo, agradezco a José Luis Falguera y C. Ulises Moulines por los comentarios y discusiones que en mucho ayudaran a llegar a la versión final. A Pablo Lorenzano agradezco de modo especial por las discusiones y su buena voluntad en ayudarme a estructurar la presentación de las ideas en el presente artículo. Ninguno de ellos son responsables de las deficiencias que aún preserve el trabajo.

Metatheoria 4(2)(2014): 9-22. ISSN 1853-2322.

(c) Editorial de la Universidad Nacional de Tres de Febrero. Publicado en la República Argentina. 


\section{Introducción}

Es ampliamente conocido que una teoría de la anomia (TA) se consolida a partir del artículo de Merton intitulado "Social Structure and Anomie" (Merton 1938). Con este trabajo, la teoría se vuelve importante y conquista su espacio, destacándose como una de las más productivas teorías acerca de la desviación, principalmente en lo se refiere al crimen, la delincuencia, la violencia, etc. Durante este período, dicha teoría pasa por algunas alteraciones y algunas "fusiones" con "otras" teorías. Este estudio se enfoca exclusivamente en la formulación de TA conforme es presentada por Merton en "Social Structure and Anomie", en tanto los desarrollos posteriores de la teoría serán objeto de estudio en trabajos ulteriores. En este artículo se exponen las principales ideas contenidas en la publicación de 1938 para posteriormente presentar la reconstrucción de TA.

\section{2. "Social Structure and Anomie"}

El artículo empieza presentando la oposición de Merton a la tendencia de los sociólogos a atribuir como causa mayor del mal funcionamiento de la estructura social los impulsos biológicos del hombre que no pueden ser socialmente controlados, perspectiva que entiende el orden social como un aparato para la gestión de impulsos y para la transformación (ablandamiento) social de las tensiones. De este modo, la no conformidad estaría basada en la naturaleza original de los individuos y la conformidad sería el fruto de su utilidad o de un condicionamento irracional. Sín embargo, esto no proporciona una base para establecer las condiciones no biológicas que llevan a las desviaciones de lo esperado socialmente en términos de conducta. En este contexto Merton afirma:

El esquema conceptual [su propuesta] que se describe está diseñado para proporcionar un enfoque coherente y sistemático para el estudio de las fuentes socio-culturales de la conducta desviada. Nuestro principal objetivo consiste en descubrir cómo algunas estructuras sociales ejercen una presión definida sobre ciertas personas en la sociedad a participar en una conducta inconformista y no [en una] conformista (Merton 1938, p. 672).

Con este propósito, Merton distingue por un lado los objetivos definidos culturalmente, que tienen el rol de un marco de referencia aspiracional y, por otro, aquello que define (controlando y regulando) los medios aceptables de tener éxito, es decir, de lograr tales metas:

Cada grupo social invariablemente acopla su escala de objetivos deseados con la regla moral o institucional de los procedimientos permitidos y requeridos para la consecución de estos fines. Estas normas regulatorias y los imperativos morales no necesariamente coinciden con las normas técnicas o de eficiencia. Muchos de los procedimientos que desde el punto de vista de los individuos particulares serían más eficientes en la obtención de los valores deseados [...] se excluyen del ámbito institucional de las conductas permitidas (Merton 1938, p. 673).

Sin embargo, aunque operen de forma conjunta, los objetivos culturales y las normas institucionales no guardan necesariamente una relación constante entre sí. Es posible que socialmente se dé mayor importancia a los objetivos que a las normas o, por otro lado, puede darse lo inverso, que las normas sean más valoradas socialmente que las objetivos [metas] culturales. Entre estos dos extremos se encuentra un número bastante grande de posibilidades. Tales posibilidades llevan a que "la conducta aberrante [...] puede ser vista como un síntoma de la disociación entre aspiraciones culturalmente definidas y medios socialmente estructurados [disponibles]" (Merton 1938, p. 674). Esto resulta en un conflicto que Merton ilustra con un fenómeno típico de la cultura estadounidense:

El énfasis extremo en la acumulación de la riqueza como un símbolo de éxito en nuestra propia sociedad se opone por completo al control efectivo de los modos de regulación institucional de la adquisición de una fortuna. El fraude, la corrupción, el vicio, la delincuencia, en fin, todo el catálogo de 
conductas prohibidas se hace cada vez más común cuando el énfasis en el origen cultural de éxitoobjetivo está divorciado de un énfasis institucional coordinado (Merton 1938, pp. 675-676).

Están, por un lado, los objetivos que culturalmente son asumidos como importantes y, por otro, los medios realmente existentes, es decir, los medios disponibles para lograr tales objetivos. Es la disociación entre ellos lo que constituye la anomia, y entonces, la presión hacia el desvío. Una vez que el individuo tiene asimilados de algún modo los objetivos socialmente valorados y las normas (la moral de la sociedad en cuestión), esta situación de conflicto hace necesaria una adaptación, que consiste en tomar una actitud, expresada por una postura frente a esta situación en lo que respecta tanto a los objetivos socialmente valorados como a los medios a la vez eficientes y aceptables para lograr tales objetivos, es decir, los medios recomendables.

Las adaptaciones son: (a) innovación, que corresponde a seguir los objetivos socialmente valorados abandonando los medios recomendables socialmente para lograr tales objetivos; (b) ritualismo, cuando se da lo inverso, es decir, el abandono de los objetivos socialmente valorados y la utilización/valoración de los medios recomendables socialmente sin la búsqueda del objetivo socialmente valorado, (c) retraimiento, que corresponde al abandono tanto de los objetivos socialmente valorados como de los medios recomendables; (d) rebelión, que además del abandono de los objetivos socialmente valorados y de los medios recomendables presupone el intento de legitimar una nueva conformación social, principalmente en lo que se refiere a los objetivos socialmente valorados y, lógicamente, también de los medios socialmente recomendables. Estas cuatro adaptaciones son conductas inconformistas. Es evidente que es posible también aceptar los objetivos socialmente valorados e intentar lograrlos a través de medios recomendables, lo que caracteriza la conducta conformista.

Cabe decir que estas adaptaciones no se refieren a rasgos de la personalidad, sino a modos de adaptación a cierta situación social, siendo posible el cambio de un tipo de adaptación a otro según sea la situación. Además, importa notar también que es la conformidad, y no la inconformidad, lo que prevalece, siendo así posible la estabilidad y continuidad de la sociedad.

La malla de las expectativas de lo que constituye todo orden social se sustenta en el modo de comportamiento de sus miembros pertenecientes a la primera categoría [conformidad]. La conducta de rol convencional orientada hacia los valores básicos del grupo es la regla y no la excepción. Es este hecho por sí solo lo que nos permite hablar de un agregado humano como constituyente de un grupo o una sociedad (Merton 1938, p. 677).

Al no obtener éxito en la búsqueda de los objetivos socialmente valorados por no tener acceso a los medios legítimos (recomendábles socialmente), es natural buscar generar una situación que permita escapar de la incómoda circunstancia de fracasar en el intento. Entre estas adaptaciones, la menos frecuente es el retraimiento. Ésta tiene lugar cuando "los objetivos de la cultura y los procedimientos institucionalizados se han asimilado completamente, es decir, con gran intensidad, por el individuo y [se han] impregnado de afecto y de alto valor positivo" (Merton 1938, p. 677), pero que, sin embargo, en la estructura social vigente, los "procedimientos institucionalizados que prometen una medida de éxito en la consecución de los objetivos no están disponibles para el individuo" (Merton 1938, p. 677). Además:

Se tomó nota que, donde la frustración se deriva de la falta de acceso a los medios institucionales eficaces para la consecución económica o de cualquer otro tipo del gran valor "éxito", las adaptaciones [...] innovación, ritualismo y rebelión tambien son posibles. El resultado será determinado por la personalidade particular, y por lo tanto, el fondo cultural particular involucrado (Merton 1938, p. 678).

En lo que se refiere a la innovación, Merton afirma que este tipo de adaptación tiene lugar cuando "la frustración se elimina por renunciar a los medios institucionales y retener la aspiración al éxito" (Merton 1938, p. 678), el ritualismo ocurre cuando "el objetivo se deja caer [...] pero la conformidad con los costumbres persiste" (Merton 1938, p. 678) y, por último, la rebelión sucede cuando "la emancipación de las normas imperantes [...] conduce a un intento de introducir 'un nuevo orden social"” (Merton 1938, p. 678), lo que significa también nuevos objetivos a ser socialmente valorados 
como más importantes. En el caso de la innovación, la baja intensidad en la asimilación de la importancia de los medios recomendables permite la renuncia a ellos, lo que no pasa con respeto a los objetivos socialmente valorados, que tienen su importáncia asimilada con más intensidad. En el caso del ritualismo lo que pasa es justamente el inverso, la asimilación es más intensa con respeto a la importancia de dichos medios que la asimilación de los objetivos socialmente valorados. La rebelión sucede cuando la asimilación de la importancia tanto de dichos objetivos como de los medios recomendables son débiles.

Con TA Merton se propone explicar la conducta desviada (inconformista) a partir de aspectos sociales (y no biológicos). Lo hace vinculando, en circunstancias específicas (la imposibilidad de acceder a los medios recomendables y el fracaso en lograr el éxito-objetivo), la asimilación de los objetivos y normas sociales con la ocurrencia de conductas desviadas. La noción clave para entender cómo Merton logra explicar la conducta desviada es la de asimilación en lo que se refiere a los objetivos socialmente valorados positivamente y a las normas (morales) vigentes en la sociedad en cuestión.

La noción de igualdad ("el sol brilla para todos"), por la cual se concibe que los objetivos socialmente valorados deben estar al alcance de todos, lleva, una vez que los medios socialmente aceptables no están de hecho al alcance de todos, a una situación en la cual "[l]a frustración y la aspiración frustrada provocan la búsqueda de vías de escape de una situación culturalmente inducida intolerable; o la ambición sin alivio puede tener como efecto intentos ilegales para adquirir los valores dominantes" (Merton 1938, p. 680).

\section{El elemento teórico básico de TA}

\subsection{La clase de los modelos potenciales de TA}

La reconstrucción de TA que aquí se presenta tiene por base la metodología de la metateoría estructuralista, conforme es presentada en An Architectonic for Science. The Structuralist Program (Balzer, Moulines \& Sneed 1987). Se presupone que el lector está familiarizado con los conceptos de dicha metateoría.

En el caso de TA, como conceptos constituyentes de la teoría están "individuos", "objetivos", "normas", "medios", "posturas". Además, está el conjunto auxiliar "símbolos comparativos". Éstos son los dominios a partir de los cuales se constituirán (conjuntistamente) las funciones "medios eficientes", "medios aceptables", "medios disponibles", "postura frente a objetivos", "postura frente a medios recomendables", "asimilar [la importancia de los] objetivos" y "asimilar [la importancia de las] reglas (normas)", además de la relación "éxito" ( $x$ logra y utilizando $z$ ). Teniendo en cuenta estos componentes conceptuales, ya es posible caracterizar el conjunto de los modelos potenciales de TA.

D1: $\quad x=\langle I, O, N, M, P, \odot$, mef, mac, mdi, pob, pmr, exi, aob, are $\rangle$ es un modelo potencial de la teoría de la anomia $\left(x \in \mathbf{M}_{\mathrm{p}}(\mathrm{TA})\right)$ syss:

(1) $I \neq \varnothing \wedge\|I\|<\aleph_{0}$ (“individuos")

(2) $\mathrm{O} \neq \varnothing \wedge\|\mathrm{O}\|<\aleph_{0}$ (“objetivos")

(3) $N \neq \varnothing \wedge\|N\|<\aleph_{0}$ ("normas")

(4) $M \neq \varnothing \wedge\|M\|<\aleph_{0}$ ("medios")

(5) $P=\left\{+,-,{ }^{*}\right\}$ ("posturas")

(6) $\odot=\{1\rfloor$,$\} (conjunto auxiliar "símbolos comparativos": variable: \chi$ ) [Conjunto auxiliar]

(7) mef: $\mathrm{O} \rightarrow(\operatorname{Pot}(\mathrm{M})-\varnothing)$ ("medios eficientes")

(8) $\operatorname{mac}: \mathrm{O} \times(\operatorname{Pot}(N)-\varnothing) \rightarrow(\operatorname{Pot}(\mathrm{M})-\varnothing)$ ("medios aceptables")

DA1: $m r e=\left\{m_{i} / m_{i} \in \mathrm{D}_{\mathrm{II}}(m e f) \wedge m_{i} \in \mathrm{D}_{\mathrm{II}}(\right.$ mac $\left.)\right\}$ ("medios recomendables")

(9) $m d i: I \times O \rightarrow \operatorname{Pot}(\mathrm{M})$ ("medios disponibles") 
(10) pob: $I \times \mathrm{O} \rightarrow P \quad$ ("postura frente a objetivos")

(11) pmr: $I \times$ mre $\rightarrow P$ ("postura frente a medios recomendables")

DA2: tco $=\left\{\left\langle\alpha, \alpha^{\prime}\right\rangle / \alpha \in \mathrm{D}_{\text {II }}(p o b) \wedge \alpha^{\prime} \in \mathrm{D}_{\text {II }}(\right.$ pmr $\left.)\right\}$ ("tipos de conductas")

(12) exi $\subseteq I \times O \times M$ ("éxito" ( $x$ logra y utilizando $z$ ))

(13) aob: $I \times \mathrm{O} \rightarrow \odot$ ("asimilar [la importancia de los] objetivos")

(14) are: $I \times(\operatorname{Pot}(N)-\varnothing) \rightarrow \odot, \operatorname{con}(\operatorname{Pot}(N)-\varnothing) \in \mathrm{D}_{\mathrm{I}}(\operatorname{mac})=(\operatorname{Pot}(N)-\varnothing) \in \mathrm{D}_{\mathrm{I}}$ (are) ("asimilar [la importancia de las] reglas (normas)") [el $\mathrm{D}_{\mathrm{I}}(\mathrm{mac})$ y el $\mathrm{D}_{\mathrm{I}}(\operatorname{are})$ no están sólo formados por (Pot(N) $\varnothing)$, sino por pares ordenados, en donde el segundo componente de dicho par es un miembro de $(\operatorname{Pot}(N)-\varnothing)$; el primero es, para mac, un elemento de $O$, y, para are, un elemento de I]

Comentario sobre este predicado:

(1) I representa el conjunto de individuos (seres humanos): personas que se relacionan con otras, formando parte de determinados contextos sociales y no de otros, inmersas en la cultura de su lugar (su lugar puede ser desde un pueblito de pescadores bastante aislado hasta el planeta como un todo, dependiendo entonces de las actividades y el nivel cultural-económico de cada individuo). Dado que TA se aplica siempre a una sociedad, se asume que el conjunto de individuos es el conjunto de individuos característico de una sociedad.

(2) O representa el conjunto de objetivos: elementos programáticos que identifican la finalidad hacia la cual deben dirigirse los recursos y esfuerzos. Pueden ser materiales (la vivienda propia) y sociales, es decir, de status (presidente de la República). También en este caso, por las razones mencionadas, se asume que el conjunto de objetivos es el conjunto de objetivos característico de una sociedad.

(3) $N$ representa el conjunto de normas: ordenamientos imperativos de acción, es decir, reglas, disposiciones o criterios para regular acciones o bien para regular los procedimientos que se deben seguir para la realización de las tareas. Del mismo modo que en el caso de los dos conjuntos anteriores, se asume éste como siendo el conjunto de normas característico de una sociedad.

(4) M representa el conjunto de medios: diligencias para conseguir algo, es decir, acciones que sirven para determinados fines. Como en los casos anteriores, se asume este conjunto como el conjunto de los medios existentes en una sociedad.

(5) P representa el conjunto de posturas. Es un conjunto constituido por las posibilidades de toma de posición que, en el marco de la teoría, cabe considerar tanto frente a los objetivos como a los medios. Las posibilidades son tres: la aceptación $(+)$, el rechazo $(-)$ y la sustitución $\left(^{*}\right)$. Como se verá de inmediato, son los valores que le corresponden al codominio de las funciones pob y pmr.

(6) $\odot$ representa el conjunto de símbolos comparativos. Es un conjunto auxiliar constituido por dos simbolos "contrarios". Por un lado, "fuerte" (representada por "1") y, por otro, "débil" (representada por “ł”). Estos símbolos serán empleados en las funciones aob y are. Trabajar con las nociones "fuerte" y "débil" parece dejar la reconstrucción un tanto imprecisa. Justamente es una suerte de imprecisión que se busca aprehender, una vez que la propia teoría esté presentada, cómo es posible notar en la descripción de los tipos de conductas inconformistas, con cierta vaguedad en la apreciación de las razones que subyacen a un tipo de conducta inconformista en específico. Los grados de asimilación no son precisados por Merton. Lo único que cuenta es que $x$ sea más o sea menos o sea de modo igual (ya sea con intensidad fuerte o débil) asimilado que $y$. Es una métrica simple para expresar una propiedad (la intensidad) de la asimilación, ya sea de normas (are) o de objetivos (aob).

(7) mef representa la función de medios eficientes. Esta función le asigna, a un determinado objetivo, un subconjunto del conjunto de los medios. La característica específica de este subconjunto de medios asignado a dicho objetivo es que estos medios son aquellos que son eficientes para lograrlo. 
(8) mac representa la función de medios aceptables. Esta función le asigna, a un determinado objetivo, frente a un subconjunto del conjunto de normas, un subconjunto del conjunto de los medios. La característica específica de este subconjunto de medios asignado a dicho objetivo es que estos medios son aquellos que son aceptables socialmente (están de acuerdo con las normas) para lograrlo.

DA1: mre representa los "medios recomendables", es decir, aquellos medios que socialmente se espera que sean utilizados para lograr los objetivos. Es un conjunto de medios que son a la vez eficientes y aceptables.

(9) mdi representa la función de medios disponibles. Esta función le asigna, a un determinado individuo, con referencia a determinado objetivo, un subconjunto del conjunto de los medios. La característica específica de este subconjunto de medios asignado a dicho individuos es que estos medios son aquellos que están disponibles para ello.

(10) pob representa la función de postura frente a objetivos. Esta función le asigna, a un determinado individuo, con referencia a determinado objetivo, una postura. Según el conjunto de posturas, tres son las posibilidades: la aceptación $(+)$, el rechazo $(-)$ y la sustitución $\left(^{*}\right)$. Por aceptación se entiende intentar lograr dicho objetivo; el rechazo significa no intentar lograrlo; la sustitución involucra el intento de reemplazo por otro objetivo a ser buscado. Esto es importante para TA una vez que la postura del individuo frente a los objetivos culturales es uno de los componentes que conforman las conductas asumidas por los individuos que la teoría se destina a explicar.

(11) pmr representa la función medios recomendables. Esta función le asigna, a un determinado individuo, con referencia a determinado(s) medio(s) recomendable(s), una postura. Del mismo modo que en pob, son tres las posibilidades: la aceptación $(+)$, el rechazo $(-)$ y la sustitución $\left(^{*}\right)$. En este caso, por aceptación se entiende utilizar (en el intento de lograr el objetivo en cuestión) algún(os) de los medios recomendables; el rechazo significa no utilizar (en el intento de lograr el objetivo en cuestión) algún(os) de los medios recomendables; la sustitución involucra el intento de reemplazar los actuales medios concebidos como recomendables por otros medios. Esto también es importante para TA una vez que la postura del individuo frente a los medios recomendables es uno de los componentes que conforman las conductas asumidas por los individuos que la teoría pretende explicar.

DA2: tco representa los "tipos de conductas". Es un conjunto de pares ordenados, con el primer elemento de dicho par perteneciendo al codominio de pob y el segundo al codominio de pmr.

(12) exi representa la relación de éxito. Es una relación entre un individuo, un objetivo y algún(os) medio(s). Representa el hecho del intento de logro de un objetivo por un individuo utilizando para ello algún medio.

(13) aob representa la función de asimilar [la importancia de los] objetivos. Esta función le asigna, a un individuo, cierta intensidad en la asimilación de la importancia de los objetivos culturales. Como se ha visto, la intensidad puede ser "fuerte" (1) o "débil" ( $)$.

(14) are representa la función de asimilar [la importancia de las] reglas (normas). Esta función le asigna, a un individuo, cierta intensidad en la asimilación de la importancia de un subconjunto del conjunto de normas culturales.

\subsection{La clase de los modelos de TA}

La clase de los modelos de una teoría son la contraparte modeloteórica de lo que en la concepción clásica de las teorías se denomina "ley". En el caso de TA, es posible expresar dicha clase del siguiente modo:

D2: $x=\langle I, O, N, M, P, \odot$, mef, mac, mdi, pob, pmr, exi, aob, are $\rangle$ es un modelo de la teoría de la anomia $(x \in$ $\mathrm{M}(\mathrm{TA}))$ syss:

(1) $x \in \mathbf{M}_{\mathrm{p}}(\mathrm{TA})$ 
(2) dados $I, O, N, M, P$ y $\odot$, existen funciones mef, mac, $m d i$, pob, pmr, aob, are y la relación exi, tales que: si mre $\cap D_{\text {II }}(m d i)=\varnothing$ y exi $=\varnothing$, entonces se cumple:

$$
(\text { aob } \neq \varnothing \wedge \text { are } \neq \varnothing)=(\text { tco } \neq\langle+,+\rangle)
$$

Comentario sobre este predicado:

(1) Expresa la condición formal de que $x$, para ser modelo de TA, pertenezca a los modelos potenciales de TA.

(2) Expresa la "ley fundamental" de TA, que afirma que, cuando existe un conflicto entre "estructura cultural y "estructura social" (representadas aquí por objetivos y normas, por un lado, y medios, por el otro), y el individuo no logra un objetivo socialmente valorado, se espera que éste presente una conducta inconformista, debido a (y según sea) la asimilación que haga de la importancia del objetivo y de las normas que regulan los modos aceptables de lograrlo.

\subsection{La clase de los modelos parciales de TA}

Con la mente puesta en la caracterización estructuralista de los modelos potenciales de una teoría, se pueden examinar los conceptos básicos de TA, a saber, I, O, N, M, P, mef, mac, mdi, pob, pmr, exi, aob, are, a fin de determinar cuáles de estos conceptos son TA-teóricos y cuáles son TA-no-teóricos, con la mente puesta en que los conceptos TA-teóricos presuponen la ley fundamental de TA para la determinación de su extensión.

En el caso de I ("individuos"), considérese la caracterización informal dada de este concepto al ser explicitados los modelos potenciales de TA: I constituye uno de los conjuntos básicos principales que establecen la ontología empírica de la teoría. Sin embargo, para averiguar si una entidad particular $i$ pertenece al conjunto I no es necesario presuponer la validez de la ley de TA; bastarían, de hecho, métodos empíricos independientes: aquellos que utilizamos para identificar observacionalmente los seres humanos y distinguirlos de las demás cosas existentes.

Lo mismo ocurre con O ("objetivos"), que también constituye otro de los conjuntos básicos principales que establecen la ontología empírica de la teoría, es decir, su determinación se da con independencia de TA. La determinación de las entidades que caen bajo este concepto puede darse por la observación del comportamiento, por encuestas y otros modos que permiten establecer la motivación para la acción, es decir, a qué está dirigido el actuar de los individuos. Por lo general, un muestreo con un número significativo de individuos es admitido por los científicos como un dato aceptable acerca de una sociedad. De este modo se puede determinar si un objetivo es característico de (valorado en) una sociedad sin presuponer TA.

En lo que se refiere a N ("normas"), que también constituye otro de los conjuntos básicos principales que establecen la ontología empírica de la teoría, la determinación de las entidades que lo constituyen es también independiente de TA. De hecho, basta observar, en la mayoría de los casos, los códigos jurídicos y los comportamientos de los individuos. La observación del comportamiento es importante en la medida en que las normas culturales actúan en un contexto que va más allá del jurídico. En las relaciones sociales puede haber sanciones a este o aquel individuo que no esté, por ejemplo, de acuerdo con el hábito, la moda, los procedimientos vigentes en determinado contexto. Como en el caso de los objetivos, por lo general, un muestreo significativo de casos individuales es admitido por los científicos como un dato aceptable acerca de una sociedad. De este modo se puede determinar si una norma es característica (conforma el aparato normativo) de una sociedad.

M ("medios"), que es también uno de los conjuntos básicos principales que establecen la ontología empírica de la teoría, comprende los modos de lograr los objetivos, es decir, las acciones que sirven para determinados fines. La determinación de las entidades que pertenecen a este conjunto se da, también, con independencia de TA. Dado un objetivo, la determinación de los medios en nada depende de TA, sino de factores, más bien técnicos, ajenos a ella. La observación basada en el 
conocimiento (concepción) que se tiene de lo que sea un medio, basta para la determinación de la extensión del concepto.

P ("posturas"), que también constituye otro de los conjuntos básicos principales que establecen la ontología empírica de la teoría, comprende las posibilidades de toma de posición frente, en el caso de la TA, tanto a los objetivos como a los medios. Como señalado, las posibilidades son tres: la aceptación $(+)$, el rechazo $(-)$ y la sustitución $\left(^{*}\right)$. Es evidente que para determinar que alguién tiene una actitud frente algo no hace falta pressuponer la TA, sino que basta el conocimiento comum que es utilizado cotidianamente en las relaciones humanas.

En el caso de $\odot$, dado que es un conjunto auxiliar, no tiene sentido aplicarle la distinción TAteórico/TA-no-teórico. Así se llega a que, entre los dominios básicos de TA, no se encuentra un concepto que sea TA-teórico. En lo que sigue, se analizan las funciones y la relación definidas (conjuntistamente) a partir de tales dominios.

En lo que respecta a mef ("medios eficientes"), determinar que un medio sea o no eficiente para lograr un determinado objetivo es independiente de TA. Más bien depende de la concepción de eficiencia. El medio será considerado eficiente si por él se logra el objetivo en cuestión. Acá, como bien apunta Merton, lo importante son factores técnicos que pueden ser aplicados, en términos de la determinación de la extensión del concepto, por la observación, teniendo en cuenta los factores de eficiencia considerados por los científicos (o, en este caso, técnicos).

En el caso de mac ("medios aceptables"), la determinación de los medios que son aceptables para lograr algún objetivo se da también de modo independiente de TA. Determinar si un determinado medio es aceptable o no, requiere averiguar si éste está o no de acuerdo con las normas en cuestión. Basta tener acceso a los códigos jurídicos (en el caso de normas legales) o a la moral (hábitos) en cuestión y entonces constatar el acuerdo o desacuerdo. En el caso de normas jurídicas la determinación se hace efectiva por medio del uso de "técnicas jurídicas" y cuando se trate de normas no formales cabe la observación acerca de la aprobación moral respecto de la utilización de este o aquel medio para lograr determinado objetivo.

En cuanto a mdi ("medios disponibles"), determinar si un medio está disponible a cierto individuo no requiere presuponer la ley TA. A partir del análisis de la condición social del individuo se puede determinar qué medios tiene él a su disposición en lo que se refiere a un determinado objetivo. En caso de que la simple observación no alcance, es bastante probable que las encuestas sociales (es decir, las encuestas gubernamentales y de los demás organismos que tratan de indicadores sociales) sirvan para determinar cuáles son las posibilidades existentes (disponibles) para cada tipo de individuo.

En el caso de pob ("postura frente a los objetivos"), la determinación de la postura es independiente de TA. Basta, de hecho, observar el comportamiento de los individuos y así averiguar si aceptan, rechazan o intentan sustituir los objetivos.

Con pmr ("postura frente a los medios recomendables") pasa lo mismo, la determinación de la postura es independiente de TA. Basta, también, observar el comportamiento de los individuos y así averiguar si aceptan, rechazan o intentan sustituir los medios recomendables.

En lo que respecta a exi ("éxito" ( $x$ logra y utilizando $z)$ ), determinar si esta relación se da o no, requiere procedimientos independientes de TA, como la observación, el sondeo o la entrevista en profundidad, o incluso la técnica denominada "focus group". En los casos en que se disponga de acceso relevante a las acciones, se puede llegar a la determinación sólo por la observación. Cuando esto no es posible, las demás técnicas surgen como alternativas.

En lo que respecta a aob ("asimilar [la importancia de los] objetivos"), en donde se le asigna al individuo una intensidad de asimilación de los objetivos culturales, parece no haber manera de determinar los valores que asume esta función sin tener en cuenta la actitud del individuo frente a los objetivos, o sea, su conducta. De este modo, una vez que la ley de la teoría afirma que la conducta (compuesta de dos actitudes, una referente a los objetivos sociales) es dependiente de la asimilación de los objetivos culturales y las normas culturales, está claro que la determinación de los valores de aob es 
dependiente de la teoría, es decir, aob es un concepto TA-teórico. No hay otro modo de determinar el valor que asume esta función en cada aplicación de TA.

También en lo que respecta a are ("asimilar [la importancia de las] reglas (normas)"), en donde se le asigna al individuo una intensidad de asimilación de las normas culturales, parece no haber manera de determinar los valores que asume esta función sin tener en cuenta la actitud del individuo frente a las normas. De este modo, una vez que la ley de la teoría afirma que la conducta (compuesta de dos actitudes, una referente a los medios recomendables) es dependiente de la asimilación de los objetivos culturales y las normas culturales, como en el caso de aob, está claro que la determinación de los valores de are es dependiente de la teoría, es decir, are es una función TA-teórica. No hay otro modo de determinar el valor que asume esta función en cada aplicación de TA.

Sabiendo que aob y are son los únicos conceptos TA-teóricos, ya se puede caracterizar la clase de los modelos parciales de TA.

D3: $y=\langle I, O, N, M, P, \odot$, mef, mac, mdi, pob, pmr, exi $\rangle$ es un modelo parcial de la teoría de la anomia $(y \in$ $\left.\mathbf{M}_{\mathrm{pp}}(\mathrm{TA})\right)$ syss existe una $x$ tal que:

(1) $x=\langle I, O, N, M, P, \odot$, mef, mac, mdi, pob, pmr, exi, aob, are $\rangle \in \mathbf{M}_{\mathrm{p}}(\mathrm{TA})$

(2) $y=\langle I, O, N, M, P, \odot$, mef, mac, mdi, pob, pmr, exi $\rangle$.

\subsection{Las condiciones de ligadura de TA}

Las restricciones impuestas por las leyes no son el único tipo de restricciones que puede imponérsele a los modelos potenciales. Otro tipo de restricciones son lo que se denomina condiciones de ligadura. Éstas vinculan modelos de una teoría según ciertas condiciones (se podría decir, nómicas), entendiéndose así como "leyes sobre leyes". En caso de que un concepto conserve invariablemente una característica de una aplicación de la teoría a otra es posible establecer una condición que exprese dicha conservación, vinculando así distintos modelos (aplicaciones) de dicha teoría.

Está claro que no se espera que un individuo tenga siempre la misma adaptación, es decir, frente al hecho de no lograr un objetivo, presente una constancia en el tipo de reacción ante dicho fracaso. Que esto no pasa ya nos lo advierte Merton al señalar que aquí no se trata de un rasgo de personalidad que nos avale esperar siempre lo mismo. Ahora bien, ya cuando uno se enfoca en las funciones TA-teóricas, $a o b$ y are, parece que sucede algo distinto. Frente a un objetivo, la asimilación del individuo es la misma (es siempre igual) en la mayoría de los casos en los contextos en que estén involucrados el mismo objetivo y el mismo individuo, para el caso de aob. Con are pasa lo mismo respecto de las normas. Aquí "en la mayoría de los casos" significa que no siempre, pero casi siempre, ocurre que la intensidad de la asimilación de un individuo con respecto a un objetivo es la misma. Una vez más, esto sirve también para are. No es "siempre" así, sino que lo es "en la mayoría de los casos", por el hecho de que puede ocurrir que, debido a la vivencia del individuo (y aquí no se utiliza el término vivencia de modo técnico, y se debe entender vivencia como llegar a la madurez, aprender algo nuevo, pasar por alguna dificultad, alcanzar un logro muy significativo, etc.) él puede cambiar el modo como asimila objetivos y/o normas. Piénsese, como un ejemplo, en el caso en que la sociedad del individuo se encuentre de pronto involucrada en un conflicto bélico. Es de esperar que los objetivos considerados hasta allí como más importantes vean bastante reducida su importancia, mientras que otros objetivos pasen a ocupar la cima de la jerarquía de importancia.

Sin embargo, no existiendo ningún factor de peso interviniendo, existe cierta regularidad a lo largo del tiempo, aun cuando con pequeñas variaciones, en el modo como el individuo asimila un objetivo. Así, pues, se asume que TA presenta dos condiciones de ligadura (ambas de igualdad), una para aob y otra para are. Esto significa que, en el caso de aob, ocurre que, cada vez que se tienen dos modelos potenciales de TA y que se dé que ambos tienen el mismo individuo y el mismo objetivo, la asimilación de este objetivo por el individuo será igual, es decir, presentará la misma intensidad. Lo que ocurre en are es análogo a lo que ocurre en aob, solamente cambiándose el objetivo por un conjunto de normas. A estas condiciones de ligadura se le asocian la cláusula ceteris paribus de que el 
individuo no sufre ningún cambio (como, por ejemplo, los arriba mencionados). La justificación para postular estas condiciones de ligadura es que, aun cuando se sepa que a lo largo de una vida uno cambia mucho en términos morales, es posible (y de hecho uno lo hace todo el tiempo) asumir que el individuo es constante en la asimilación del objetivo y/o de las normas.

Cabe notar que la condición de ligadura de identidad de aob es independiente de la condición de ligadura de identidad de are. No requiere mucho esfuerzo encontrar casos en que, en lo que respecta a ciertos objetivos, las reglas a seguir para lograrlo de modo aceptable hayan cambiado a lo largo del tiempo. Tampoco requiere esfuerzo encontrar casos en donde la normas sirven para regular el modo aceptable de lograr más de un objetivo.

D4: La condición de ligadura de identidad de $a o b$ " $\mathbf{C}_{a o b}^{(\approx)=\rangle}$ " (en donde el subíndice indica la función de la que se trata, a saber, la función $a o b$, y el supraíndice el tipo de condición de ligadura, a saber, de igualdad), está definida por $X \in \mathbf{C}_{a o b}^{(\approx)=>}$ si y sólo si $X \subseteq \mathbf{M}_{\mathrm{p}}(\mathrm{TA})$ y para toda combinación posible $x$, $x^{\prime} \in X \subseteq \mathbf{M}_{\mathrm{p}}(\mathrm{TA})$, en donde $x=\langle I, \mathrm{O}, \mathrm{N}, \mathrm{M}, P, \odot$, mef, mac, mdi, pob, pmr, exi, aob, are $\rangle$ y $x^{\prime}=\left\langle I^{\prime}, \mathrm{O}^{\prime}\right.$, $N^{\prime}, M^{\prime}, P^{\prime}, \odot$ ', mef', mac', mdi', pob', pmr', exi', aob', are' $\rangle$, se cumple que:

$$
\langle i, o, \chi\rangle \in\left(I \cap I^{\prime}\right) \times\left(O \cap O^{\prime}\right) \times\left(\odot \cap \odot^{\prime}\right) \rightarrow\left(a o b(i, o, \chi) \leftrightarrow a o b^{\prime}(i, o, \chi)\right)
$$

D5: La condición de ligadura de $a o b$ " $\mathbf{C}_{a r e}^{(\tilde{z},=)}$ " (en donde el subíndice indica la función de la que se trata, a saber, la función are, y el supraíndice el tipo de condición de ligadura, a saber, de igualdad), está definida por $X \in \mathbf{C}_{\text {are }}^{\langle\approx \text { re }}$ si y sólo si $X \subseteq \mathbf{M}_{\mathrm{p}}(\mathrm{TA})$ y para toda combinación posible $x$, $x^{\prime} \in X \subseteq \mathbf{M}_{\mathrm{p}}$ (TA), en donde $x=\langle\mathrm{I}, \mathrm{O}, \mathrm{N}, \mathrm{M}, P, \odot$, mef, mac, mdi, pob, pmr, exi, aob, are $\rangle$ y $x^{\prime}=\left\langle I^{\prime}, \mathrm{O}^{\prime}\right.$, $N^{\prime}, M^{\prime}, P^{\prime}, \odot$ ', mef', mac', mdi', pob', pmr', exi', $a b^{\prime}$, $\left.a e^{\prime}\right\rangle$, se cumple que:

$$
\begin{aligned}
& \left\langle i, n_{l(l \in \mathbb{N} \wedge 1 \leq l \leq n)}, \chi\right\rangle \in\left(I \cap I^{\prime}\right) \times\left(N \cap N^{\prime}\right) \times\left(\odot \cap \odot^{\prime}\right) \rightarrow \\
& \quad\left(\operatorname{are}\left(i, n_{l(l \in \mathbb{N} \wedge 1 \leq l \leq n)}, \chi\right) \leftrightarrow \operatorname{are}^{\prime}\left(i, n_{l(l \in \mathbb{N} \wedge 1 \leq l \leq n)}, \chi\right)\right)
\end{aligned}
$$

El efecto constrictivo conjunto de las condiciones ligaduras de TA, es decir, su condición de ligadura global GC(TA), se expresa mediante la intersección conjuntista de las dos condiciones específicas: $\mathrm{GC}(\mathrm{TA})=\mathbf{C}_{a a b}^{(\tilde{\approx}, \Rightarrow\rangle} \cap \mathbf{C}_{\text {are }}^{\langle\approx \approx=\rangle}$.

\subsection{Los vínculos interteóricos de TA}

En lo que se refiere a los vínculos interteóricos, no existen para el caso de las funciones aob ("asimilar [la importancia de los] objetivos") y are ("asimilar [la importancia de las] reglas (normas)"), pues son conceptos TA-teóricos. Veamos qué se puede decir acerca de los demás conceptos presentes en la teoría. Cabe notar que, como en cierto sentido ya señala la discusión acerca de los métodos de determinación de los conceptos TA-no-teóricos, la teoría no tiene vínculo alguno con alguna teoría científica cristalizada, es decir, plasmada en el conocimiento científico de modo de estar presente en libros de textos, de ser enseñada en las universidades a las nuevas generaciones, etc. Se verá que hay vínculos, pero éstos son con el conomimiento de sentido común, pre-teorías, proto-teorías o folktheories. ${ }^{1}$ El propio Merton no indica de dónde provienen los conceptos que utiliza en la teoría. A continuación se trata, entonces, de explicitar, en la medida de lo posible, tales vínculos interteóricos.

Comencemos con I ("individuos"). Este concepto procede de alguna "teoría" proveniente del conocimiento común; con esta "teoría" sería posible establecer la interpretación intencional básica de este conjunto base principal. Simbolizemos este vínculo por medio de $\lambda_{1}$.

Algo similar ocurre con $O$ ("objetivos"). El concepto de objetivo proviene también de alguna "teoría" del conocimiento común, o algo un poco más elaborado (proto-teoría), con la cual también sería posible establecer la interpretación intencional básica del conjunto de objetivos. Simbolizemos este vínculo por medio de $\lambda_{2}$.

\footnotetext{
${ }^{1}$ Para una discusión detallada, véase Lorenzano (2013).
} 
A través de N ("normas"), TA se vincula con alguna teoría de la normativización, aun cuando ésta no sea demasiado elaborada o detallada. Podríamos pensar en una teoría que ya encontramos en posesión por parte de aquellos que tienen que liderar grupos, que trabajan en gestión de instituciones (escuelas, ejército, empresas, etc.,...). Simbolizemos este vínculo por medio de $\lambda_{3}$.

En el caso de M ("medios"), hay una larga literatura acerca de los medios (y su relación con los fines) que viene desde los antiguos griegos. La noción de medio es extremadamente conocida y forma parte del conocimiento previo existente. Simbolizemos este vínculo por medio de $\lambda_{4}$.

En cuanto a $P$ parece no existir una teoría desarrollada acerca de las posturas (actitudes) aceptación, del rechazo y la sustitución, las que son relevantes para la teoría. En este caso el vínculo interteórico sería con el conocimiento perteneciente al sentido común. Simbolicemos este vínculo por medio de $\lambda_{5}$.

Por medio de $\odot$ ("símbolos comparativos"), se podría postular un vínculo interteórico con alguna teoría de la medición o de la comparación (en la medida en que esta última es distinta de la primera). Sin embargo, se trata en este caso de un conocimiento de sentido común o del uso bastante relajado de una teoría en sentido estricto. Simbolizemos este vínculo por medio de $\lambda_{6}$.

Tratándose de mef ("medios eficientes"), TA podría vincularse con alguna "teoría de la gestión". Sin embargo, parece ser que el uso del concepto está vinculado con un conocimiento práctico que ya se tiene. Se apela al entendimiento común compartido acerca de lo que son medios y eficiencia. Simbolizemos este vínculo por medio de $\lambda_{7}$.

En lo que respecta a mac ("medios aceptables"), sucede exactamente lo mismo que con los medios eficientes: el uso del concepto está con un conocimiento práctico que ya se tiene. Se apela al entendimiento común compartido acerca de lo que son medios y lo que es aceptable en lo que se refiere a ellos. Simbolizemos este vínculo por medio de $\lambda_{8}$.

En cuanto a mdi ("medios disponibles"), TA se vincula con alguna teoría de la disponibilidad. Parece ser, también en este caso, que se trata de un entendimiento práctico de lo que es estar disponible. Simbolizemos este vínculo por medio de $\lambda_{9}$.

Por medio de pob ("postura frente a los objetivos"), función que representa la actitud de un individuo frente a objetivos socialmente aceptados, TA se vincula con el conocimiento perteneciente al sentido común que afirma la capacidad de reacción del individuo frente a algo. También para este caso, Merton no indica aquí ninguna teoría presupuesta específica. Sin embargo, es importante para TA que se asuma que frente a los objetivos socialmente valorados el individuo presente cierta actitud (una de las tres que son relevantes para la teoría). Simbolizemos este vínculo por medio de $\lambda_{10}$.

Con pmr ("postura frente a los medios recomendables"), la función que a un individuo le asigna una actitud frente a los medios recomendables, pasa exactamente lo mismo que con pob: por medio de esta función la teoría se vincula con el conocimiento perteneciente al sentido común que afirma la capacidad de reacción del individuo frente a algo. Tampoco aquí indica Merton alguna teoría específica presupuesta, pero evidentemente también en este caso es importante para TA que se asuma que frente a los medios aceptables socialmente el individuo presente cierta actitud (también aquí, una de las tres que son relevantes para la teoría). Simbolizemos este vínculo por medio de $\lambda_{11}$.

En lo que respecta a exi ("éxito" ( $x$ logra y utilizando $z$ )), relación que representa el supuesto de que los individuos logran los objetivos socialmente valorados por medio de la utilización de ciertos medios, no hay nada en lo que dice Merton que haga referencia a una teoría del éxito. Asumamos también aquí que la interpretación intencional básica viene dada por el conocimiento común. Simbolizemos este vínculo por medio de $\lambda_{12}$.

El vínculo interteórico global de TA (L(TA)), formado por la intersección de todos los vínculos interteóricos que tiene esta teoría con otros saberes subyacentes (presupuestos), se define de la siguiente manera:

D6: $\mathrm{L}(\mathrm{TA}) \stackrel{\text { def }}{=} \cap\left\{\lambda_{1}, \lambda_{2}, \lambda_{3}, \lambda_{4}, \lambda_{5}, \lambda_{6}, \lambda_{7}, \lambda_{8}, \lambda_{9}, \lambda_{10}, \lambda_{11}, \lambda_{12}\right\}$. 


\subsection{Las aplicaciones intencionales de la TA}

El dominio de aplicaciones intencionales de TA está constituido por la clase de aquellos sistemas empíricos a los que se desea aplicar la ley fundamental de dicha teoría. Estos sistemas no pueden ser caracterizados por medios puramente formales y, además, lo único que se puede decir desde el punto de vista formal es que $\mathbf{I}(\mathrm{TA}) \subseteq \mathrm{M}_{\mathrm{pp}}(\mathrm{TA})$. Entre los posibles sistemas empíricos que satisfacen dicha condición formal, cuáles pertenecen al conjunto de las aplicaciones intencionales de una teoría lo deciden, con el auxilio de las aplicaciones paradigmáticas, ${ }^{2}$ los miembros de la comunidad científica pragmáticamente. En el caso de TA algunos ejemplos de estos sistemas, que pueden extraerse del texto del propio Merton, son:

En una forma de conducta desviada, los individuos se atienen a las metas ensalzadas por la cultura mientras que abandonan los medios aprobados por ella para tratar de lograrlos. Naturalmente este esquema no es específico de una sociedad que proclama altas aspiraciones para todos. Pero una sociedad tal tiende a esfumar la línea demarcatoria entre los caminos legítimos e ilegítimos para la autopromoción, puesto que lo que cuenta más que nada es el resultado. Hecha esta composición de lugar, no nos sorprenderá el descubrimiento de los sociólogos de Cornell de que en las universidades americanas los estudiantes más consagrados al "éxito monetario" y a "progresar en el mundo", sostienen, mucho más a menudo que los otros, que "no pueden permitirse el lujo de tener remilgos en cuanto a los medios" que utilicen.

Un segundo tipo de respuesta se da cuando hombres que han internalizado las normas morales a tal punto que no puedan ser violadas, se refugian en la rutina. Abandonan a disgusto sus aspiraciones mientras pueden, tal vez con más firmeza aún a causa de ello, de la rutina de sus roles. Son los ritualistas de nuestra sociedad, incontados aunque probablemente legión: los autómatas de la organización, los rutinarios, los religiosos compulsivos.

Otros, en fin, se retraen completamente de la lucha por asegurarse un lugar, abandonando tanto sus esperanzas como la relativa seguridad de cumplir de modo rutinario con las reglas. Están en la sociedad, pero no son de ella. Ésos son los retraídos que juntan una vida de a pedacitos, comiendo un poco, durmiendo mucho y abandonando el esfuerzo de crear pautas de la vida cotidiana que puedan respetar. En lo esencial son pasivos, llevan una existencia semioculta (hote-and-carner existence), enajenados de la gama más amplia de los valores sociales. Algunos, por último, consiguen aniquilar al mundo matándose. Naturalmente éstos no son todos los tipos de respuesta desviada. Muy significativos son los rebeldes quienes, al igual que los retraídos, repudian toda una gama de metas y medios culturalmente prescritos pero que, a diferencia de aquéllos, tratan en forma deliberada de desarrollar otros nuevos, teniendo sus propias pretensiones de legitimidad. A ellos pertenecen los disidentes que apelan a una moralidad superior, fundándose en los valores últimos más bien que en las normas particulares de la sociedad. (Merton 1964, pp. 218-219)

Teniendo en cuenta los modelos parciales de TA $(y=\langle I, O, N, M, P, \odot$, mef, mac, mdi, pob, pmr, exi $\rangle)$ es posible expresar lo presentado por Merton en términos de aplicaciones intencionales de TA, es decir, utilizando los términos TA-no-teóricos. Sea, por ejemplo, una conducta de innovación.

- ¿Por cuál razón se constata que los estudiantes [I], para progresar en el mundo [O], aun conociendo el reglamento [N] de la universidad, con relación a los medios [M], en caso de que no tengan disponibles [mdi] medios que sean a la vez aceptables [mac] y eficientes [mef], y por lo tanto les sea imposible lograr el objetivo [exi] de modo aceptable socialmente, muy bien podrían intentar lograrlo $[p o b=+]$ utilizando medios no recomendables [pmr $=-$ ]?

De modo similar, también pueden ser descritos en términos de los modelos parciales de TA los otros ejemplos dados por Merton, aun cuando resulte más trabajoso hacerlo.

\footnotetext{
${ }^{2}$ Las aplicaciones paradigmáticas son aquellas aplicaciones exitosas que se convierten en casos-tipo o típicos de nuevas aplicaciones de la teoría. Las aplicaciones paradigmáticas constituyen las instancias concretas en donde se muestra la conexión entre la teoría y la investigación empírica.
} 


\section{Las especializaciones del elemento teórico básico de TA}

La red teórica de TA presenta un único nivel de especialización con cuatro líneas. Estas especializaciones se dan de acuerdo con las restricciones impuestas al conjunto de las asimilaciones hechas por los individuos de los objetivos y de las normas culturales, es decir, al conjunto definido a partir de los valores asumidos por las funciones aob y are, ambas funciones TA-teóricas.

\subsection{Primera línea de especialización}

La primera línea de especialización restringe la asimilación a que el individuo haya asimilado con mayor intensidad los objetivos que las normas culturales.

D7: $x=\langle I, O, N, M, P, \odot$, mef, mac, mdi, pob, pmr, exi, aob, are $\rangle$ es un modelo de la teoría de la anomia para la innovación $(x \in \mathrm{M}(\mathrm{TAINN}))$ syss:

(1) $x \in \mathrm{M}(\mathrm{TA})$

(2) $\mathrm{D}_{\mathrm{II}}(a \circ b)=1 \wedge \mathrm{D}_{\mathrm{II}}($ are $\left.)=\right\rfloor=($ tco $=\langle+,-\rangle)$

Comentario sobre este predicado:

(1) Expresa la condición formal de que $x$, para ser modelo de TAINN, debe pertenecer a los modelos de TA.

(2) Expresa la ley especial, a saber, la afirmación de que, cuando un individuo asimila más la importancia del objetivo que la de las normas, presenta una conducta innovadora.

\subsection{Segunda línea de especialización}

La segunda línea de especialización restringe la asimilación a que el individuo haya asimilado con mayor intensidad las normas que los objetivos culturales.

D8: $x=\langle I, O, N, M, P, \odot$, mef, mac, mdi, pob, pmr, exi, aob, are $\rangle$ es un modelo de la teoría de la anomia para el ritualismo $(x \in \mathrm{M}(\mathrm{TARIT}))$ syss:

(1) $x \in \mathrm{M}(\mathrm{TA})$

(2) $\mathrm{D}_{\mathrm{II}}(a \circ b)=\downarrow \wedge \mathrm{D}_{\mathrm{II}}($ are $)=\uparrow=($ tco $=\langle-,+\rangle)$

Comentario sobre este predicado:

(1) Expresa la condición formal de que $x$, para ser modelo de TARIT, pertenezca a los modelos de TA.

(2) Expresa la ley especial, a saber, la afirmación de que cuando asimila más la importancia de las normas que la del objetivo, el individuo presenta una conducta ritualista.

\subsection{Tercera línea de especialización}

La tercera línea de especialización restringe la asimilación a que el individuo haya asimilado igualmente, y con fuerte intensidad, los objetivos y las normas culturales.

D9: $x=\langle I, O, N, M, P, \odot$, mef, mac, mdi, pob, pmr, exi, aob, are $\rangle$ es un modelo de la teoría de la anomia para el retraimiento $(x \in \mathrm{M}(\mathrm{TARET}))$ syss:

(1) $x \in \mathrm{M}(\mathrm{TA})$

(2) $\mathrm{D}_{\mathrm{II}}(a \circ b)=1 \wedge \mathrm{D}_{\mathrm{II}}($ are $)=1=($ tco $=\langle-,-\rangle)$

Comentario sobre este predicado:

(1) Expresa la condición formal de que $x$, para ser modelo de TARET, pertenezca a los modelos de TA. 
(2) Expresa la ley especial, a saber, la afirmación de que cuando asimila fuertemente tanto la importancia de las normas como la del objetivo, el individuo presenta una conducta de retraimiento.

\subsection{Cuarta línea de especialización}

La cuarta línea de especialización restringe la asimilación a que el individuo haya asimilado igualmente, y con poca intensidad, los objetivos y las normas culturales.

D10: $x=\langle I, O, N, M, P, \odot$, mef, mac, mdi, pob, pmr, exi, aob, are $\rangle$ es un modelo de la teoría de la anomia para la rebelión ( $x \in \mathrm{M}(\mathrm{TAREB}))$ syss:

(1) $\quad x \in \mathrm{M}(\mathrm{TA})$

(2) $\mathrm{D}_{\mathrm{II}}($ aob $)=1 \wedge \mathrm{D}_{\mathrm{II}}(\operatorname{are})=1=\left(\right.$ tco $\left.=\left\langle{ }^{*},{ }^{*}\right\rangle\right)$

Comentario sobre este predicado:

(1) Expresa la condición formal de que $x$, para ser modelo de TAREB, pertenezca a los modelos de TA.

(2) Expresa la ley especial, a saber, la afirmación de que cuando asimila de modo débil tanto la importancia de las normas como la del objetivo, el individuo presenta una conducta de rebelión.

\section{La red teórica de TA}

Enunciadas las especializaciones de la teoría, es posible expresar gráficamente la red teórica de TA del siguiente modo:

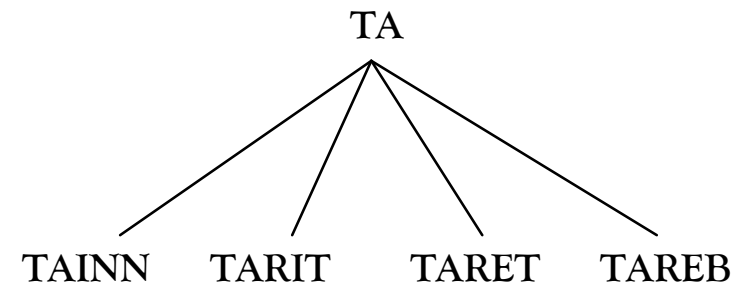

Figura 1. Representación gráfica de la red teórica de TA

Bibliografía

Balzer, W., Moulines, C.U. y J.D. Sneed (1987), An Architectonic for Science. The Structuralist Program, Dordrecht: Reidel. (Traducción castellana y edición revisada de Pablo Lorenzano: Una arquitectónica para la ciencia. El programa estructuralista, Bernal: Universidad Nacional de Quilmes, 2012.)

Clinard, B.M. (ed.) (1964), Anomie and Deviant Behavior: A Discussion and Critique, Nueva York: Free Press.

Lorenzano, P. (2013), “Aspectos erotéticos del «hibridismo» de Mendel”, Contrastes. Revista Internacional de Filosofía 18: $451-468$.

Merton, R.K. (1938), "Social Structure and Anomie”, American Sociological Review 3: 672-682.

Merton, R.K. (1964), “Anomie, Anomia, and Social Interaction: Contexts of Deviant Behavior”, en Clinard (1964), pp. 213-242. 